\title{
AUTOMATIC GENERALIZABILITY METHOD OF URBAN DRAINAGE PIPE NETWORK CONSIDERING MULTI-FEATURES
}

\author{
Shaonan Zhu', ${ }^{1, *}$, Qiqi Yang ${ }^{2}$, Jiaqi Shao ${ }^{2}$ \\ ${ }^{1}$ College of Geographical and Biological Information, Nanjing University of Posts and Telecommunications, Nanjing, China - \\ zhushaonan@njupt.edu.cn \\ ${ }^{2}$ School of Geography Science, Nanjing Normal University, Nanjing, China
}

Commission VI, WG VI/4

KEY WORDS: Stroke, Urban Drainage Pipe Network, Generalization, Similarity Criterion, Multi-features

\begin{abstract}
:
Urban drainage systems are indispensable dataset for storm-flooding simulation. Given data availability and current computing power, the structure and complexity of urban drainage systems require to be simplify. However, till data, the simplify procedure mainly depend on manual operation that always leads to mistakes and lower work efficiency. This work referenced the classification methodology of road system, and proposed a conception of pipeline stroke. Further, length of pipeline, angle between two pipelines, the pipeline belonged road level and diameter of pipeline were chosen as the similarity criterion to generate the pipeline stroke. Finally, designed the automatic method to generalize drainage systems with the concern of multi-features. This technique can improve the efficiency and accuracy of the generalization of drainage systems. In addition, it is beneficial to the study of urban storm-floods.
\end{abstract}

\section{INTRODUCTION}

It is one of the major global issues that storm-flooding hazards have taken place increasingly frequent and serious with the increase of extreme climate events (Hammond et al., 2015; Duan et al., 2016). The accurate simulation and prediction of urban storm-flooding, and on this basis, putting forward countermeasures is an effective measure to reduce urban stormfloods (Croci et al., 2014). The simulation of the urban surface faces enormous challenges, which contains many factors, such as rainfall intensity, subcatchments, geomorphology, etc. (Yu et al., 2015). Hence, the quantity and quality of the data determine the simulation precision. The drainage pipe network is an indispensable factor in the division of urban confluence units. The complexity of the drainage pipe network determines the complexity of catchment units (Alcaraz et al., 2009). The rainwater well, rainwater gate and rainwater inlet play a role of catchment while pipes play a role in drainage. Urban drainage systems are indispensable dataset for storm-flooding simulation (Park et al., 2008). Considering the complexity of the structure of drainage pipe network, the lager amount of involving datasets and the wide coverage area, the survey is difficult. The generalization of rainwater pipe network has great influence on the morphology of subcatchments, and the complexity of the rainwater pipe network determines the complexity of catchment unit. Traditional rainstorm waterlogging is usually structural and spatial simplified before modelling given data availability and current computing power (Ghosh et al., 2012; Krebs et al.,2014). However, at this stage, most of the simplified or generalized methods of drainage pipe network use artificial means and rely on experience to delete some pipeline data, which is inefficient and error-prone.

In this paper, a comprehensive automation method of urban drainage pipe network is proposed to improve the efficiency and accuracy of pipe network generalization. The urban drainage pipe network is similar to the road network, and its spatial structure, attribute information and shape feature are obvious.
Hence, the road network matching algorithm has some reference to the matching of underground pipelines. Stroke which represents a linear element connected by an extension of the intersection has smooth and consistent features and also is widely used in urban structure and road map generalization (Zhou, 2012; Ren et al., 2013). This work references the classification methodology of road system, and proposes the conception of pipeline stroke.

\section{METHODOLOGY}

\subsection{Feature Analysis of Pipe Network}

The underground pipe network is an important infrastructure of city, known as urban lifeline engineering. Pipe network is composed of pipe line sections and subsidiary facilities, and is a system constituted by tree or reticular formation. Generally, the pipeline network is provided with three characteristics: geometry, attribute and semantics. In terms of geometry, pipe network consists of pipe point and pipe section, and the connection of the pipe point and the tube segment is inseparable. It has a typical "node-arc" structure that has obvious spatial features, and the nodes are relatively dense. Pipe network has abundant attributes, such as material, depth, length, etc. Furthermore, Pipe network has unique semantics, different types of pipe points have different meanings, such as valve, exit point, repair well and so on.

In summary, the pipe network and the road network are similar in many aspects. However, different from the road data, the types of nodes in the underground pipeline are diverse, and the nodes are distributed densely. In addition, nodes or arcs which in the pipeline data have more complex semantic information than that in road network data. Therefore, considering the spatial structure and strong connectivity of the pipe network, the generalization method of road map can't be referred directly. There is some error in using the single geometric distance to measure the pipeline similarity from the view of the arc. 
So, the set of pipe network stroke can be defined as key parts of pipe network, and they can maintain the geometry, attribute and semantics characteristics of pipe network.

\subsection{Generation Algorithm of Pipe Network Stroke}

Pipe Network Stroke is a network structure composed of similar elements in the pipeline network. While generalizing the shape of underground pipelines, it can better maintain the shape and distribution of pipelines. The process of generating pipeline Stroke is to generate new pipe segment which is formed by connecting similar segments, it has well connectivity and ductility. In Figure 1, (b) shows a pipe network Stroke sample, it has less segments compared to the original data (a).

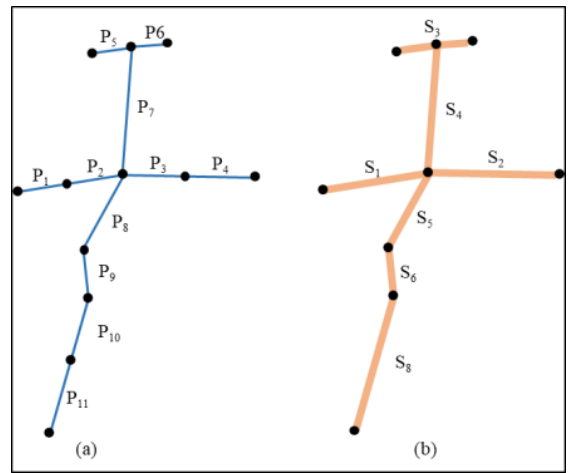

Figure 1. Pipe and Stroke Network Sample

The key issue of pipe network stroke is how to union pipe segments. The rules should be based on two aspects of geometry and semantic, such as the direction of pipe segments needs to be consistent, two pipe segments can be connected while the angle is close to $180^{\circ}$ or they have same name, etc. So the generation process of pipe network stroke can be designed, in Figure 2.
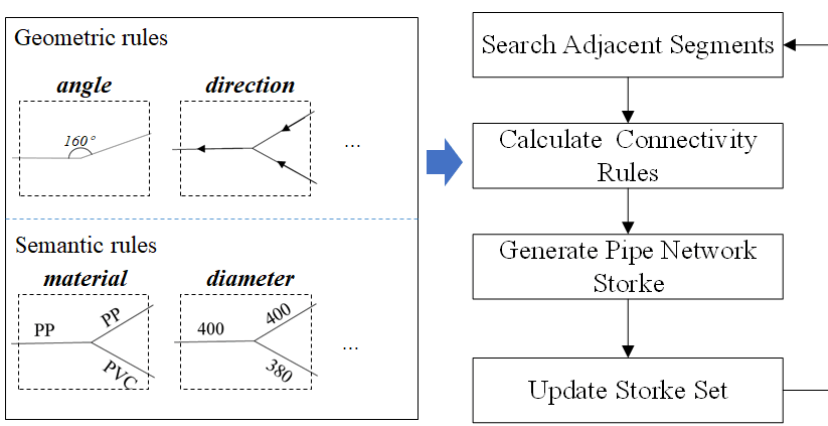

Figure 2. Generation Process of Pipe Network Stroke

Stroke pipeline is generated by classifying $\mathrm{N}$ pipelines in to $\mathrm{M}$ strokes $(\mathrm{N} \geq \mathrm{M})$, it can be formalized as: $\mathrm{P}$ is the set of pipelines in original data, $\mathrm{P}=\left\{\mathrm{P}_{1}, \mathrm{P}_{2}, \ldots, \mathrm{P}_{\mathrm{N}}\right\}$, and $\mathrm{S}$ is the set of stroke pipeline, $S=\left\{S_{1}, S_{2}, \ldots, S_{M}\right\}$.

Step1: To eliminate erroneous data by Topology processing of pipe network data.

Step2: Classify all pipe sections into stroke pipe subsets $\left(\mathrm{S}_{1}\right)$ and non-stroke pipe sections $\left(S_{2}\right)$. Select $S_{2}$ from the seed pipe section $\mathrm{P}_{1}$, and judge whether there is connectivity with other pipe sections connected with starting and ending points of $\mathrm{P}_{1}$.

Step3: If there is, then the pipe section is similar to the seed pipe section. If a similarity value of the pipe section $P_{n}$ is higher than a given threshold value, the pipe section and the seed pipe section are merged into a stroke, and classify $P_{n}$ into $S_{1}$.

Step4: Do the recursive actions to classify every pipe section into particular stroke.
The calculation of connectivity rules combined multi-features, which contained length of pipeline, angle between two pipe lines, the pipeline belonged road level and diameter of pipeline. Length of pipeline can be set according to the actual situation.

\subsection{Evaluation Significance of Pipe Network Stroke}

After calculating the stroke connectivity, the next step is stroke importance evaluation. Length, connectivity, connectivity, centrality and other stroke features are selected, and on the basis of their normalization and the corresponding weight relationship to get the value of stroke. There are some notable points: the importance of pipelines belonged main roads is higher than that of secondary roads, the pipelines have higher the importance when they have higher connectivity.

\section{EXPRIMENTAL AND RESULTS}

\subsection{Datasets}

The experimental area selected was downtown of Lishui City in Zhejiang Province, in Figure 3. Lishui is in the southeastern part of China, has moderate urban size. The total entries of the pipeline reached 4400 , and the total length of the pipeline is more than 77042 meters. In the dataset, minimum is less than $1 \mathrm{~m}$, and the maximum is $117 \mathrm{~m}$. If the original data was used to simulate storm-floods, redundant data will cause huge amount of computation.

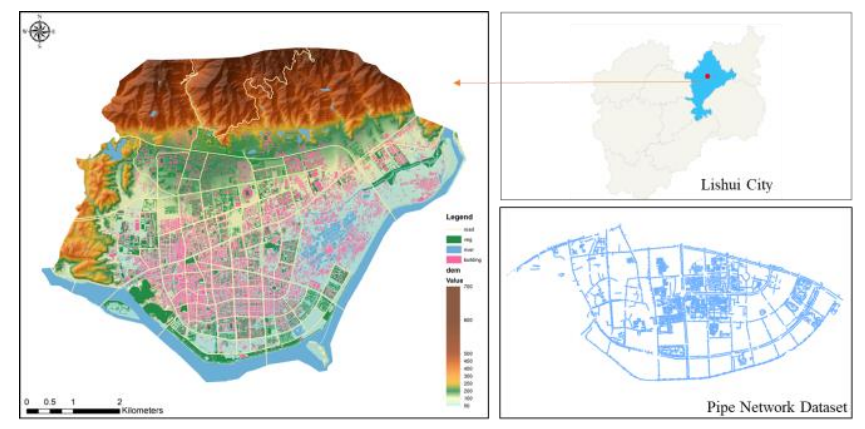

Figure 3. Experimental Area

\subsection{Generated Pipe Network Stroke}

According to the method proposed in this study, the pipe segments was traversed successively. When connectivity was calculated between two pipe segments, geometric and semantic rules were considered, as shown in Table 1 . In order to ensure the connectivity of the pipe segments, the constraints of Multifeatures are given. The length of pipe segment is longer than 30 metres, the angle should be more than $150^{\circ}$, and the flow direction of pipe segments should be consistent, but also diameter, material, name of pipe segments and road level the pipeline belonged should be same.

\begin{tabular}{|c|c|c|}
\hline \multicolumn{2}{|c|}{ Feature } & Quota \\
\hline \multirow{3}{*}{ Geometric } & Length & $>30 \mathrm{~m}$ \\
\cline { 2 - 3 } & Angle & $150^{\circ}$ \\
\cline { 2 - 3 } Semantic & Direction & Consistency \\
\hline \multirow{3}{*}{} & Diameter & Same \\
\cline { 2 - 3 } & Material & Same \\
\cline { 2 - 3 } & Name & Same \\
\cline { 2 - 3 } & Road Level & Same \\
\hline
\end{tabular}

Table 1. Multi-features in Connectivity Rules 
The generation algorithm of pipe network stroke ran 35 seconds to get the result as shown in Figure 4, and the main configuration environment contained that CPU was Intel Core i7-7700 and RAM was $8 \mathrm{G}$. It was obvious that the result maintained the shape and distribution of pipe network. As shown in Table 2, the number of pipe segments could be found to be significantly reduced, the total length of stroke network accounted for $75 \%$ the total length of pipe network. On the other hand, the average length was also greatly improved, it substantially eliminated redundant data

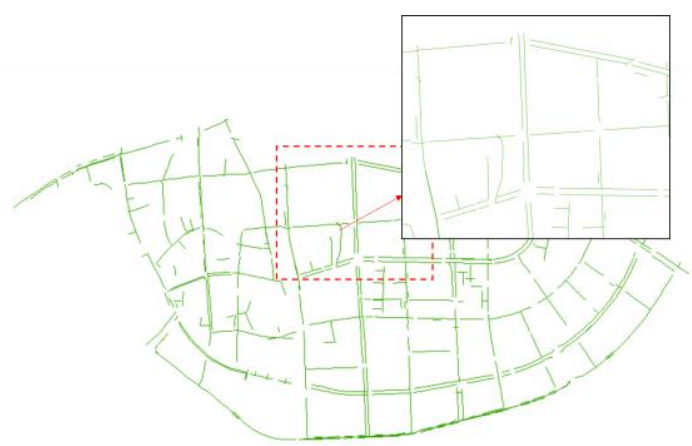

Figure 4. Experimental Area

\begin{tabular}{|c|c|c|c|c|c|}
\hline & Count & Sum(m) & Max $(\mathrm{m})$ & Mini(m) & Mean(m) \\
\hline Pipelines & 4400 & 77042.78 & 117.78 & 0.016 & 17.501 \\
\hline Strokes & 342 & 58107.31 & 1829.64 & 33.03 & 169.90 \\
\hline
\end{tabular}

Table 2. Comparison of Experimental Calculation

In the above algorithm, the initial seed was randomly selected. Piping Tee is characteristic pipe point. Further, Piping Tee points were selected to be tested, the total length of stroke obtained was 42293.16 metres, and the total amount was 156 . Half of the amount account for more than $70 \%$ of the original length. As shown by experiments, the efficiency of the algorithm improved by setting the characteristic pipe points as seeds. Generating strokes by using pipe points with morphological features illustrated that the generalizability of the pipe network should give consideration to the characteristics of the pipeline entity

\section{CONCLUSION}

In summary, this work proposed a conception of pipeline stroke chosen length of pipeline, angle between two pipelines, the pipeline belonged road level and diameter of pipeline as the similarity criterion to generate the pipeline stroke, and designed the automatic method to generalize drainage systems with multi-features. Experiments show that this technique highly improved the efficiency and accuracy of the generalization of drainage systems. In addition, it is of great significance to rainstorm and flood research in urban. Also, the automatic generalizability method of urban pipe network can be widely used in pipe network matching. In the future work, how to search the characteristic points of the pipe network ingeniously is the key work of this study.

\section{ACKNOWLEDGEMENTS}

Supported by National Natural Science Foundation of China (Grant No. 41501438) and NUPTSF (Grant No. NY214198).

\section{REFERENCES}

Hammond, M.J., Chen, A.S., Djordjević, S., Butler, D., \& Mark, O., 2015. Urban flood impact assessment: A state-of-the-art review. Urban Water Journal, 12, pp. 14-29.

Duan W, He B, Nover D, et al., 2016. Floods and associated socioeconomic damages in China over the last century. Natural Hazards, 82(1), pp. 401-413.

Croci S, Paoletti A, Tabellini P., 2014. URBFEP Model for Basin Scale Simulation of Urban Floods Constrained by Sewerage's Size Limitations. Procedia Engineering, 70(70), pp. 389-398.

Yu D, Coulthard T J., 2015. Evaluating the importance of catchment hydrological parameters for urban surface water flood modelling using a simple hydro-inundation model. Journal of Hydrology, 524, pp. 385-400.

Alcaraz S A, Sannier C, Vitorino A C T, et al., 2009. Comparison of methodologies for automatic generation of limits and drainage networks for hidrographic basins. Revista Brasileira de Engenharia Agrícola e Ambiental - Agriambi, 13(4), pp. 369-375.

Park S Y, Lee K W, Park I H, et al., 2008. Effect of the aggregation level of surface runoff fields and sewer network for a SWMM simulation. Desalination, 226(1), pp. 328-337.

Ghosh I, Hellweger F L., 2012. Effects of Spatial Resolution in Urban Hydrologic Simulations. Journal of Hydrologic Engineering, 17(1), pp.129-137.

Krebs G, Kokkonen T, Valtanen M, et al., 2014. Spatial resolution considerations for urban hydrological modelling. Journal of Hydrology, 512(9), pp. 482-497.

Ren C, Fang H, Lei Y, et al., 2015. Network functionality oriented stroke building in road networks. International Conference on Geoinformatics, 126, pp. 1-6.

Qi Zhou, Zhilin Li., 2012. A comparative study of various strategies to concatenate road segments into strokes for map generalization. International Journal of Geographical Information Science, 26(4), pp. 691-715. 